\title{
Les guides de pratique et les conflits d'intérêts
}

$\mathrm{E}$ n 2003, un comité d'experts chargé des guides de pratique de l'Association canadienne du diabète (ACD) a recommandé que l'on utilise le nouvel agent à effet prolongé, l'insuline glargine, comme substitut de l'insuline à effet prolongé générique pour le traitement du diabète de type I et II chez les patients qui arrivent mal à contrôler glycémie à jeun ou qui font de l'hypoglycémie nocturne ${ }^{1}$. Cet automne, des membres du Programme commun d'évaluation des médicaments (PCEM), processus consultatif national dans le cadre duquel on évalue les médicaments pour les formulaires provinciaux, ont recommandé de ne pas inscrire le médicament $^{2}$. Les deux groupes d'experts ont évalué à peu près les mêmes données probantes tirées d'une vingtaine d'essais contrôlés randomisés. Les lignes directrices de l'ACD n'indiquent pas si les membres de son groupe d'experts avaient des liens financiers de quelque nature que ce soit avec le fabricant de l'insuline glargine et, au moment d'aller sous presse, l'Association n'avait pas été en mesure de nous fournir ces renseignements. Des trois experts engagés par le PCEM, un avait été rémunéré pour donner des conférences d'éducation et rédiger un article pour le fabricant de l'insuline glargine.

Pour répondre à la recommandation du PECM, l'ACD a publié une lettre ouverte aux ministres provinciaux de la Santé (voir www .diabetes.ca/Section_Advocacy/adv_resources.asp) pour exprimer de "sérieuses préoccupations" au sujet des recommandations du PCEM et notamment souligner le fait que le groupe du PCEM ne comptait «aucun expert des médicaments contre le diabète».

La controverse soulevée par les lignes directrices ne touche pas seulement celles de l'ACD. Des débats semblables ont eu lieu au sujet de la prise charge de l'hypertension, où les lignes directrices nationales recommandent des médicaments brevetés coûteux plutôt que les produits génériques éprouvés. Presque tous les groupes de rédaction de guides et de lignes directrices de consensus reçoivent l'appui d'entreprises intéressées, et de nombreux membres de ces groupes reçoivent de ces mêmes entreprises des subventions de recherche et des honoraires pour prononcer des conférences et donner des conseils.

Le problème est profond et étendu. Dans un récent rapport portant sur plus de 200 guides (provenant de divers pays) déposés en 2004 au National Guideline Clearinghouse des ÉtatsUnis, ont constate que "plus du tiers des auteurs ont déclaré avoir des liens financiers avec les sociétés pharmaceutiques en cause et environ $70 \%$ des groupes sont touchés ${ }^{3}$. De plus, presque la moitié des guides ne disaient rien des conflits d'intérêts.

Soutenir que de tels conflits de nature financière n'ont aucun effet sur les recommandations des membres des groupes, c'est ne pas tenir compte des preuves du contraire qui s'accumulent. Une recommandation portant sur un guide national qui est enI tachée par l'argent sous forme d'honoraires de conférence et de consultation, d'actions, d'options, de brevets et de redevances, peut réussir à grossir le chiffre d'affaires et les bénéfices des entreprises, mais peut aussi nuire aux patients. Et l'on prescrira presque toujours des médicaments plus coûteux. (Selon le PCEM, l'insuline glargine coûte cinq fois plus cher que l'insuline à effet prolongé générique.)
La divulgation des conflits d'intérêts est généralement acceptée par les journaux médicaux réputés. Même si la divulgation des conflits d'intérêts d'ordre financier devrait éveiller le doute chez les lecteurs, elle ne libère pas les auteurs des guides des effets de leurs liens financiers avec l'industrie, qui pourraient être compromettants. Même si les liens en question ne leur imposent pas d'obligation, on croira certainement que c'est le cas.

Les recommandations d'experts sans expertise clinique sontelles partiales elles aussi, comme le laisse entendre l'ACD? Cela nous semble moins probable, particulièrement lorsque les experts en question évaluent des données probantes tirées d'études cliniques. Même si l'expertise clinique est importante dans la conception d'une étude (surtout lorsqu'il s'agit de tirer des conclusions cliniquement significatives), l'interprétation des résultats nécessite de l'expertise en conception et analyse d'études et pas nécessairement en pratique clinique.

Face aux preuves de plus en plus nombreuses montrant que les conflits d'intérêts d'ordre financier font pencher les recommandations des experts en faveur des produits des commanditaires, le Journal (tout comme la plupart des principaux journaux médicaux) n'acceptera pas pour publication des énoncés de consensus, critiques narratives, commentaires et articles semblables qui recommandent des médicaments, appareils, examens de laboratoire ou autres interventions et à l'égard desquels au moins un des auteurs se trouve en situation de conflit d'intérêts d'ordre financier important ${ }^{4}$.

Jusqu'à maintenant, nous avons exclu les guides de cette politique en exigeant seulement la divulgation publique des conflits d'intérêts d'ordre financier. Nous remettons maintenant cette politique en question. Entre-temps, nous demanderons aux rédacteurs de guide de nous faire parvenir une divulgation détaillée de leurs conflits d'intérêts avant de nous soumettre leur manuscrit. Ces renseignements seront communiqués par la suite aux examinateurs pairs si l'on envisage de publier le document.

Les associations nationales existent pour défendre les intérêts des patients. Elles doivent trouver un moyen d'appuyer la création de guides sans compter sur le financement d'entreprises qui ont des intérêts acquis. Entre-temps, les créateurs de guide doivent tout de même divulguer aux lecteurs, non initiés et professionnels, les conflits d'intérêts d'ordre financier des experts qui formulent des recommandations. Les médecins, les patients et les contribuables ne méritent pas moins. - JAMC

\section{RÉFÉRENCES}

I. Comité d'experts des lignes directrices de pratique clinique de l'Association canadienne du diabète. Lignes directrices de pratique 2003 de l'Association canadienne du diabète pour la prévention et le traitement du diabète au Canada. Can J Diabetes 2003;27(suppl. 2) :SI-SI62. Disponible à : www.diabetes.ca/cpgfrancais/down loads/cpgcomplete.pdf (consulté le 3I octobre 2005).

2. Office canadien de coordination de l'évaluation des technologies de la santé. CEDAC final recommendation on reconsideration and reasons for recommendation. Insulin glargine (Lantus ${ }^{\circledR}$ - Aventis Phrama Inc.). 2005. www.ccohta.ca/CDR/cdr_pdf /cdr_submissions/Complete/cdr_complete_Lantus_2005Sept28.pdf

3. Taylor R, Giles J. Cash interests taint drug advice. Nature 2005;437:I070-I.

4. Conflits d'intérêts et placements [éditorial]. JAMC 2004;I7I(II) : I315. 